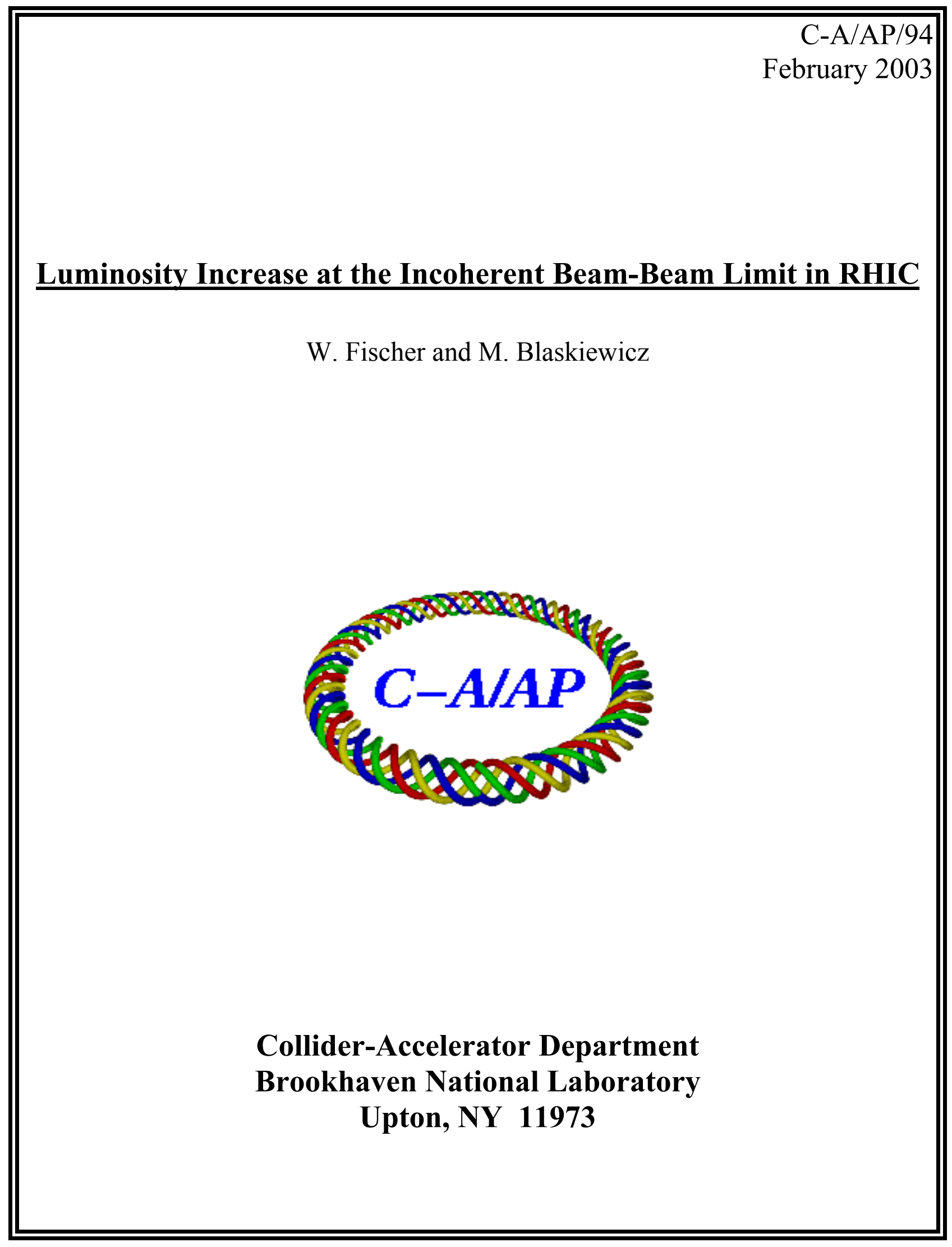


BNL C-AD/AP/94

\title{
Luminosity Increase at the Incoherent Beam-Beam Limit in RHIC
}

\author{
W. Fischer and M. Blaskiewicz
}

February 27, 2003

\begin{abstract}
By colliding bunches of greater length under a larger angle, the tune spread caused by the beambeam interaction can be reduced. Assuming a constant limit for the beam-beam tune drift, the bunch intensity can then be increased. In this way, a luminosity increase is possible. We review this strategy for proton beams in RHIC with two collisions. We consider short bunches in the storage rf system, long bunches in the accelerating rf system, and superbunches that fill the whole circumference except for an abort gap. Resonances driven by the beam-beam interaction and coherent effects are neglected in this article.
\end{abstract}

\section{Introduction}

Luminosity limits set by the incoherent beam-beam tune shift were discussed for unbunched beams by Keil [1]. He showed that an increase in the crossing angle reduces the beam-beam tune shift and allows a higher line density, which in turn leads to an increased luminosity. Recently, Ruggiero and Zimmermann extended this analysis to bunched beams [2]. They computed the incoherent beam-beam tune shift as a function of crossing angle and bunch length and showed how the luminosity increases at the beambeam limit with an increase in the crossing angle or bunch length. With one horizontal and one vertical collision under the same angle, the beam-beam tune spread in both planes is the same.

Extremely long bunches, called superbunches, are the basis of recently proposed hadron collider concept [3]. In this proposal, beam is stacked in very long bunches using barrier cavities, and accelerated with an induction device [4].

In this article we investigate the effects of bunch length and crossing angle on the achievable luminosity in RHIC proton-proton operation, given a constant incoherent beam-beam limit. We consider three cases: bunches in the storage rf system, bunches in the accelerating rf system, and superbunches. In Tab. 1 initial parameters for three reference cases are listed. The bunched cases (Gaussian short and Gaussian long) are consistent with recent running conditions and expected improvements. The rms bunch lengths for the two Gaussian cases is different by a factor of three. For the superbunch case we assume that $4 \cdot 10^{11}$ protons can be accelerated in 112 bunches and then transferred into a superbunch. A barrier bucket keeps $10 \%$ of the circumference free of beam to provide an abort gap. Neglecting end effects, this describes the collisions of coasting beam. 
Table 1: RHIC parameters for the calculation of incoherent beam-beam tune shifts and luminosities.

\begin{tabular}{lcccc}
\hline \hline quantity & unit & $\begin{array}{c}\text { Gaussian } \\
\text { short }\end{array}$ & $\begin{array}{c}\text { Gaussian } \\
\text { long }\end{array}$ & Superbunch \\
\hline incoherent beam-beam limit $\Delta Q_{\max }$ & $\ldots$ & & -0.03 & \\
lattice $\beta^{*}$ & $\mathrm{~m}$ & & 1.0 & \\
beam relativistic $\gamma$ & $\ldots$ & & 260 & \\
transverse emittance $\epsilon_{N}, 95 \%$ & $\mu \mathrm{m}$ & & 20 & \\
interaction region length $l$ & $\mathrm{~m}$ & & 20 & \\
effective detector length $l_{\text {det }}$ & $\mathrm{m}$ & & 0.7 & \\
particles per bunch $N_{b}$ & $10^{11}$ & 2.0 & 500 \\
number of bunches $n_{b}$ & $\ldots$ & & 112 & \\
bunch area $S, 95 \%$ & $\mathrm{eV} \cdot \mathrm{s}$ & 1.0 & $\ldots$ \\
rf frequency $f_{r f}$ & $\mathrm{MHz}$ & 197 & 28 & $\ldots$ \\
gap voltage $V_{g a p}$ & $\mathrm{MV}$ & 3.0 & 0.3 & $\ldots$ \\
rms bunch length $\sigma_{z}$ & $\mathrm{~m}$ & 0.15 & 0.45 & $\ldots$ \\
\hline \hline
\end{tabular}

We take for the length, in which the beam-beam force is active, the distance between the DX beam splitting magnets. Once the beams reach these magnets they are quickly separated. The effective detector length, the region in which collisions are recorded, is the largest length currently used by any one of the RHIC detectors [5].

The crossing angle $\theta$ is measured as the full angle from one beam to the other beam. We concentrate on a scenario with one collision in the horizontal plane and one collision in the vertical plane, both under the same angle $\theta$. RHIC allows to implement relatively large horizontal and smaller vertical crossing angles. In the 2001 Gold run, after a problem in one of the IP2 DX magnets was discovered, its current was reduced temporarily. With the reduced current a beam-to-beam crossing angle of up to $\theta=1.81 \mathrm{mrad}$ was implemented. With the current vertical corrector strength, a crossing angle of $0.84 \mathrm{mrad}$ can be implemented at storage energy [7]. However, some of this strength may be needed to correct for unwanted orbit effects. We therefore assume that vertical crossing angles of $0.5 \mathrm{mrad}$ can be implemented with the existing hardware.

We assume that a total tune spread of $\Delta Q_{\max }=-0.03$, caused by two collisions, can be accommodated. This is consistent with the maximum values achieved at the SPS and Tevatron. We neglect resonances driven by the beam-beam interaction and coherent effects.

\section{Beam-Beam Tune Shifts}

In Ref. [2] formulas are given for the incoherent tune shift due to the beam-beam interaction for particles in the beam center. In Fig. 1 these tune shifts are plotted as a function of a crossing angle for the cases listed in Tab. 1. In parts (a), (c) and (e) the horizontal and vertical tune shifts are depicted for a single crossing in the horizontal plane and the three cases under investigation. In parts (b), (d) and (f) the total tune shifts are shown for two crossing, one in the horizontal plane and one in the vertical plane, both under the same angle $\theta$. 

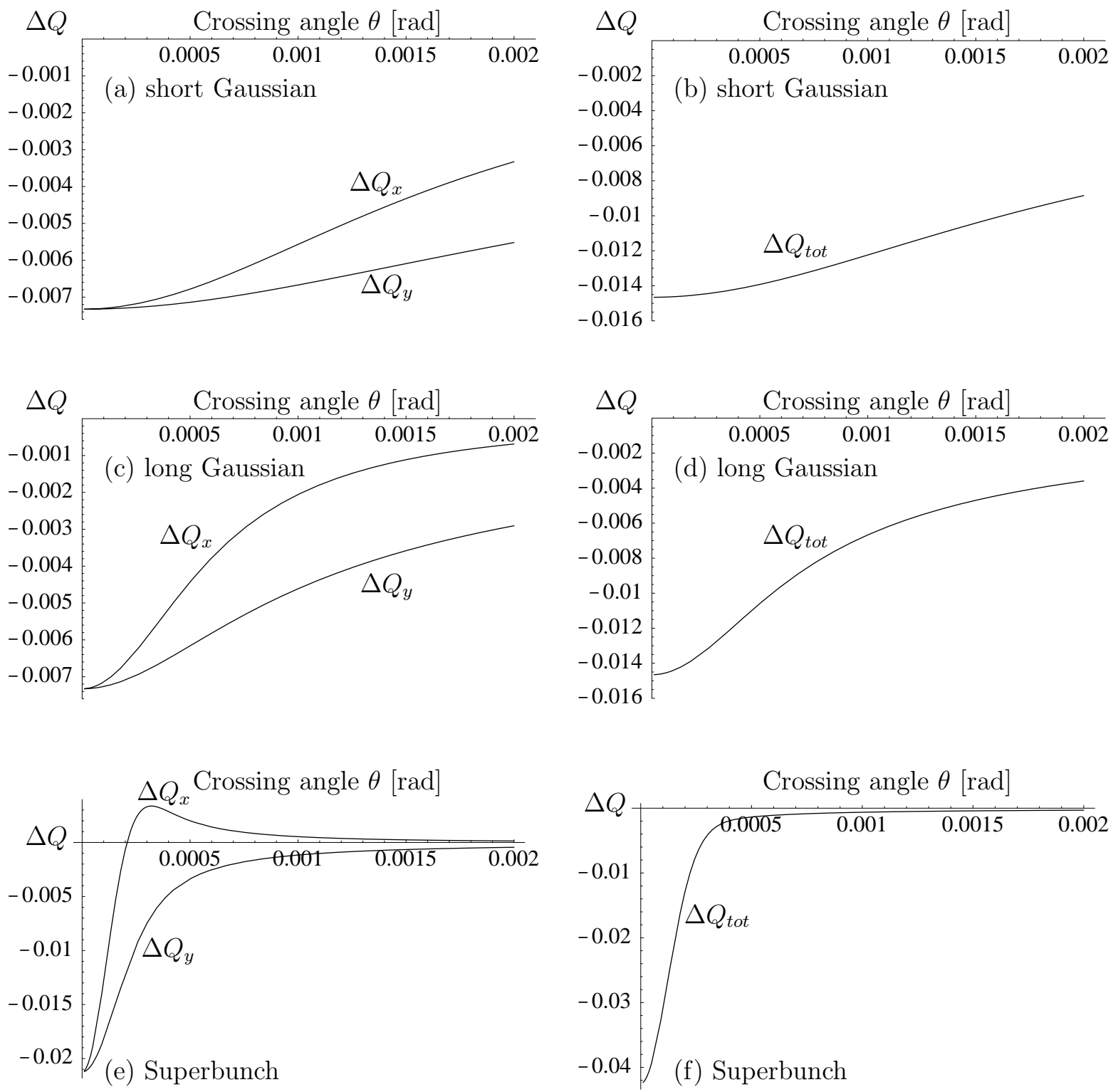

Figure 1: Incoherent beam-beam tune shifts as a function of crossing angle. Beam parameters are given in Tab. 1. Parts (a), (c) and (d) show the horizontal and vertical tune change for a single horizontal crossing. Parts (b), (e) and (f) show the total tune shift in either plane for two crossings, each under an angle $\theta$, assuming one crossing in the horizontal plane and one in the vertical plane. 
For a single crossing the values of the beam-beam tune shift are reduced in both planes with increasing crossing angle. The reduction is larger in the crossing plane than in the plane orthogonal to it. The tune shift reduction is more pronounced for the longer bunches.

For superbunches the tune shift even changes sign in the crossing plane, for large enough crossing angles. In this case, the long-range beam-beam interactions dominate over the head-on interactions. Long-range interactions produce a tune shift that has the opposite sign of the head-on tune shift.

With a crossing angle of $0.5 \mathrm{mrad}$, which can be implemented with the existing hardware, and two crossings in alternating planes, there is only a small effect for short Gaussian bunches in the storage rf system. For bunches in the accelerating rf system, the beambeam tune shift is reduced by about one third, and for superbunches by about $90 \%$.

\section{Luminosities}

In Fig. 2 (a) the luminosity per crossing is shown as a function of the crossing angle for the two bunched cases of Tab. 1. For the short bunches the luminosity without crossing angle is about 25\% larger than for the long bunches and decreases somewhat slower with an increasing crossing angle. The luminosity of superbunches with the parameters of Tab. 1, shown in Fig. 2 (b), is significantly lower than for the bunched cases.

Parts (c) and (d) depict the number of particles at which the beam-beam limit of $\Delta Q_{\max }=-0.03$ is reached, assuming two collisions in alternating planes. The longer the bunches the more particles can be put in a bunch before the limit is reached. Note that in the past only about $10^{11}$ protons per bunch were accelerated in 55 bunches. Also note, that we assume a constant transverse emittance and bunch area. In reality there will be some increase in these quantities with intensity.

In parts (e) and (f) the luminosities at the beam limit are shown. For short Gaussian bunches only small increases are possible. For long Gaussian bunches crossing angles larger than $0.5 \mathrm{mrad}$ are needed to achieve luminosities larger than those achievable with short bunches. At large crossing angles, bunch intensity and luminosity increase linearly with the crossing angle.

For two collisions an incoherent beam-beam limit of $\Delta Q_{\max }=-0.03$ allows a luminosity of the order of $10^{33} \mathrm{~cm}^{-2} s^{-1}$ for bunched beams, and of $10^{34}-10^{35} \mathrm{~cm}^{-2} \mathrm{~s}^{-1}$ for superbunches, provided that the required intensities can be accelerated and held in the machine. At the beam-beam limit the achievable luminosity is proportional the the bunch intensity and the beam-beam tune shift $\Delta Q_{\max }$. It is not dependent on the emittance since both the beam-beam tune shift and the luminosity are inversely proportional to the emittance. 
Luminosity $\left[\mathrm{cm}^{-2} \mathrm{~s}^{-1}\right]$

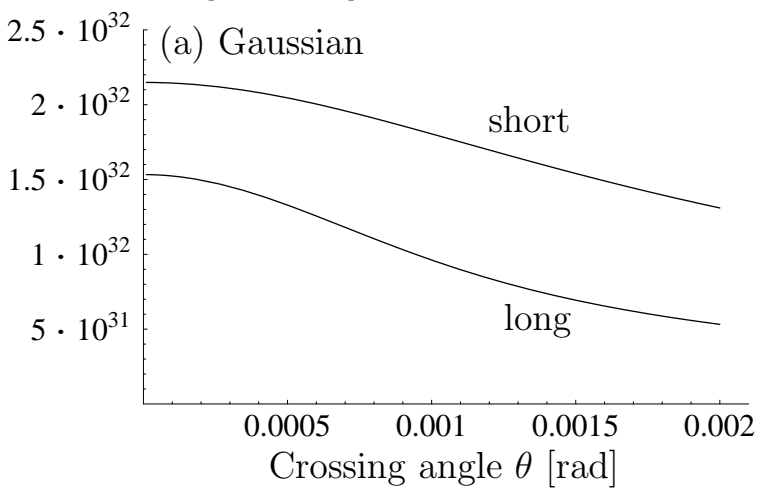

Particles per bunch $N_{b}$

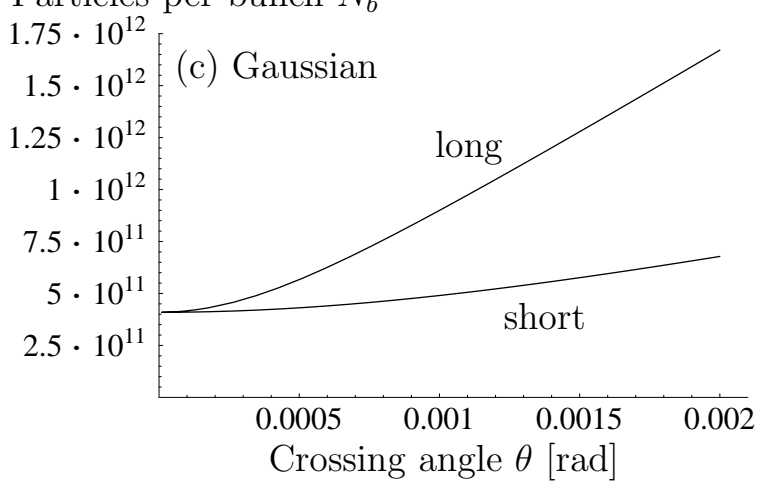

Luminosity $\left[\mathrm{cm}^{-2} \mathrm{~s}^{-1}\right]$

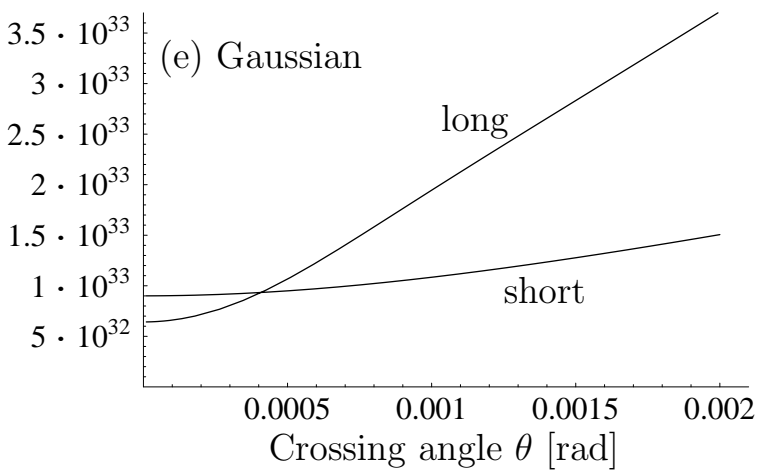

Luminosity $\left[\mathrm{cm}^{-2} \mathrm{~s}^{-1}\right]$

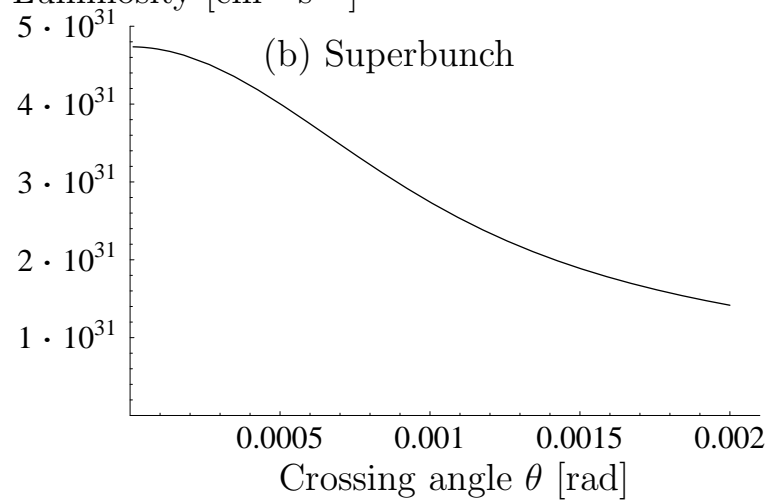

Particles per superbunch $N_{b}$

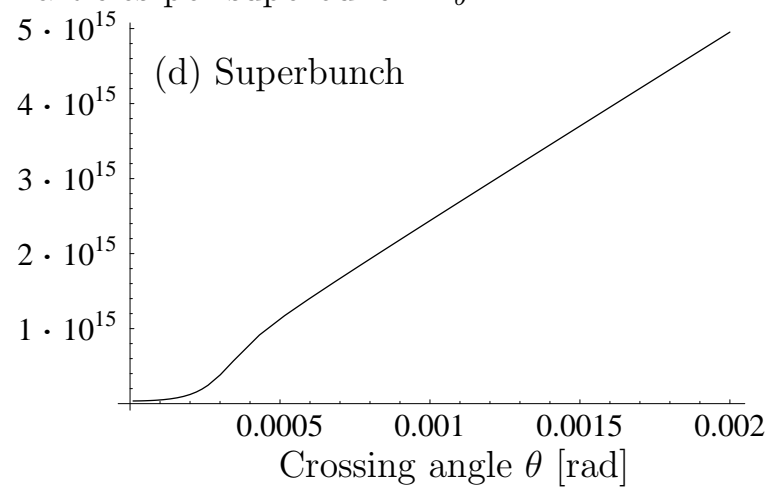

Luminosity $\left[\mathrm{cm}^{-2} \mathrm{~s}^{-1}\right]$

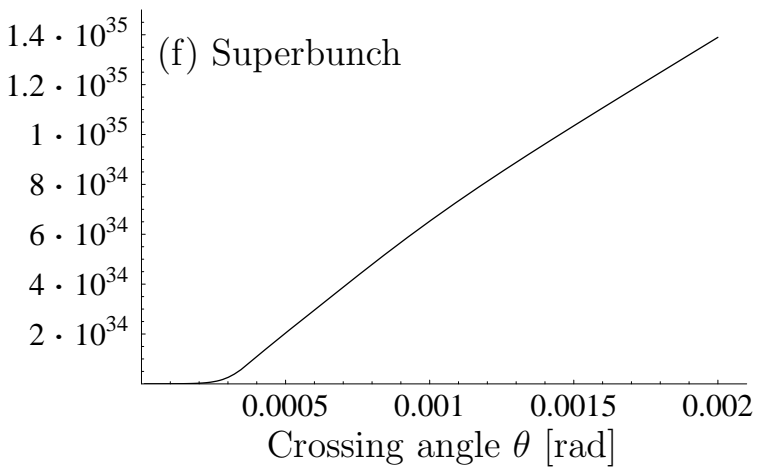

Figure 2: Luminosity per crossing as a function of crossing angle in parts (a) and (b), with beam parameters given in Tab. 1. One horizontal and one vertical crossing under the same angle $\theta$ is assumed. Parts (c) and (d) show the number of particles per bunch at the incoherent beam-beam limit of $\Delta Q_{\max }=$ -0.03 . Part (e) and (f) depict the luminosity at the beam-beam limit. 
For small Gaussian bunches and crossing angles one has [2]

$$
\begin{gathered}
L=\frac{f_{\text {coll }} \gamma}{2 r_{p}} \frac{N_{b}}{\beta^{*}}\left|\Delta Q_{\text {max }}\right| \\
\left(\theta \ll 1, \sigma_{z} \ll \beta^{*}, \sigma_{z} \ll l_{\text {det }}, \sigma^{*} \ll \sigma_{z}\right)
\end{gathered}
$$

where $f_{\text {coll }}$ is the collisions frequency, $r_{p}$ the classical proton radius, and $\sigma^{*}$ the rms beam size at the interaction point. Plots of the luminosity dependence on a number of parameters, including bunch length and crossing angle, can also be found in Ref. [8].

\section{Superbunch Gap Maintenance and Acceleration}

The highest luminosities can be achieved with superbunches. However, this requires a large number of stored protons. With $10^{15}$ protons as shown in Fig. 2 (d) the beam current is about $12 \mathrm{~A}$, comparable to the ISR beam current [9]. If particles can be accelerated and stored in buckets, the luminosity after debunching will always be lower than in the the bunched case. In this section the maintenance of an abort gap and the possibility of acceleration in the nearly debunched mode, using barrier cavities, is discussed. Experience with and plans for barrier cavities are reported in Refs. [4,10-12]. An alternative acceleration scheme is based on induction devices $[3,4]$.

Let $\epsilon=E-E_{s}$ denote the energy deviation for a given particle and let $\tau$ denote its arrival time with respect to the synchronous particle. Using turn number $n$ as the time-like variable the equations for $\tau$ and $\epsilon$ are

$$
\begin{aligned}
& \frac{d \epsilon}{d n}=-q V_{s}+q V_{r f}(\tau), \\
& \frac{d \tau}{d n}=T_{r e v} \eta \frac{\epsilon}{\beta^{2} E_{s}},
\end{aligned}
$$

where $q$ is the particle charge, $V$ the rf voltage, $T_{\text {rev }}$ the revolution time, $\eta$ the slip factor and $\beta$ the relativistic beam parameter. The subscript $s$ denotes the synchronous particle. Eqs. (2) and (3) correspond to the Hamiltonian

$$
H(\tau, \epsilon)=\frac{T_{r e v} \eta}{2} \frac{\epsilon^{2}}{\beta^{2} E_{s}}+q V_{s} \tau-q \int_{0}^{\tau} V_{r f}\left(\tau_{1}\right) d \tau_{1} .
$$

For adiabatic processes the phase space density is constant on curves of constant $H(\epsilon, \tau)$. For these a dimensionless potential energy $U(\tau)$ can be defined by

$$
\frac{\epsilon^{2}}{E_{s}^{2}}+\frac{2 \beta^{2}}{\eta T_{r e v}\left(E_{s} / q\right)}\left[V_{s} \tau-\int_{0}^{\tau} V_{r f}\left(\tau_{1}\right) d \tau_{1}\right] \equiv \frac{\epsilon^{2}}{E_{s}^{2}}+U(\tau)=\frac{\hat{\epsilon}^{2}}{E_{s}^{2}},
$$




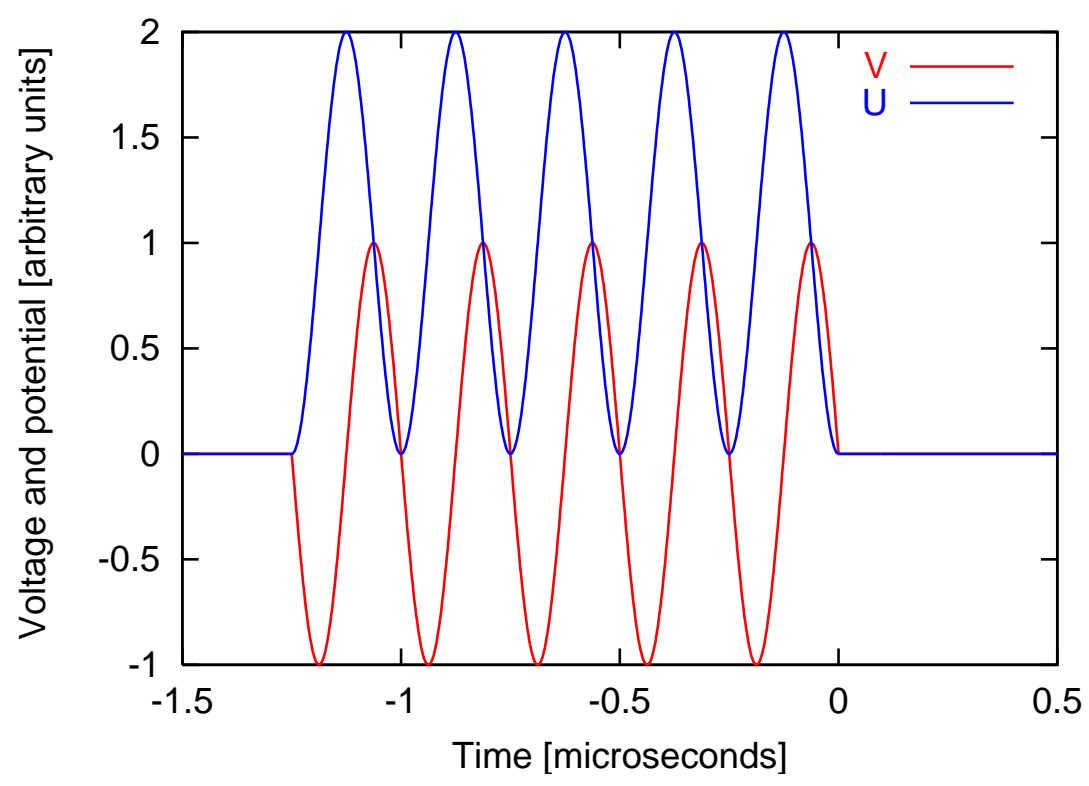

Figure 3: Voltage $V$, and potential $U$ waveforms of a barrier cavity.

where $\hat{\epsilon}=\left(E-E_{s}\right)_{\max }$ denotes the maximum energy deviation on a given contour. We choose $V_{r f}$ so that $U(\tau) \geq 0$. With Eq. (5) the potential and rf voltage for a given energy deviation $\hat{\epsilon}$ can be determined for a given waveform of the barrier cavity voltage.

Gap maintenance. In this case $V_{s}=0$. An abort gap of $1 \mu$ s length can be created, for example, by one of a $f_{r f}=1 \mathrm{MHz}$ rf system [11]. The voltage and potential waveforms for such a system are illustrated in Fig. 3, where a sinusoidal waveform for the voltage is assumed, $V(t)=-\hat{V} \sin \left(2 \pi f_{r f} t\right)$. The peak voltage $\hat{V}$ as a function of the energy spread $\hat{\epsilon}$ can be obtained from Eq. (5) as

$$
\hat{V}=\frac{\pi}{2} \frac{\eta T_{r e v} f_{r f}}{\beta^{2}} \frac{E_{s}}{q} \frac{\hat{\epsilon}^{2}}{E_{s}^{2}} .
$$

With an energy spread of $\hat{\epsilon} / E_{s}=10^{-3}$ and a frequency of $f_{r f}=1 \mathrm{MHz}$ the peak voltage needed at injection and storage is $0.2 \mathrm{kV}$ and $9 \mathrm{kV}$ respectively (see Tab Tab. 2). Previous barrier cavity work has created $10 \mathrm{kV}$ single period sine waves using a single cavity [11]. Thus gap maintenance appears possible.

Acceleration. We assume that the barrier cavity creates a high narrow barrier at $\tau=0$, and concentrate on the effect of the synchronous accelerating voltage $V_{s}$. From Eq. (5) the energy spread as a function of $\tau$ is

$$
\frac{\epsilon^{2}}{E_{s}^{2}}=\frac{\hat{\epsilon}^{2}}{E_{s}^{2}}-\frac{2 \beta^{2}}{\eta T_{r e v}\left(E_{s} / q\right)} V_{s} \tau
$$

where $0 \leq \tau \leq T_{\text {rev }}$. Setting $\tau=\lambda T_{\text {rev }}$ and $\epsilon=0$ in equation (7) gives an energy spread $\hat{\epsilon}$ which corresponds to a bunch with a current waveform given by $I(\tau) \propto \sqrt{\left(\lambda T_{\text {rev }}-\tau\right)}$. 
Table 2: Assumed rf parameters at injection and storage.

\begin{tabular}{lccc}
\hline \hline quantity & unit & injection & storage \\
\hline relativistic $\gamma$ & $\ldots$ & 26 & 260 \\
kinetic energy $E_{k}$ & $\mathrm{GeV}$ & 23.4 & 243.0 \\
slip factor $\eta$ & $\ldots$ & 0.00044 & 0.00191 \\
energy spread $\hat{\epsilon}$ & $\ldots$ & $10^{-3}$ & $10^{-3}$ \\
barrier frequency $f_{r f}$ & $\mathrm{MHz}$ & 1.0 & 1.0 \\
$\hat{V}$ for abort gap maintenance & $\mathrm{kV}$ & 0.2 & 9 \\
\hline \hline
\end{tabular}

For $\lambda=1$ the bunching factor is $2 / 3$. The required energy spread is

$$
\frac{\epsilon_{0}}{E_{s}}=\sqrt{\frac{2 \lambda V_{s}}{\eta\left(E_{s} / q\right)}}
$$

Taking $\lambda=1, \eta=10^{-4}, V_{s}=12 \mathrm{kV}$ and $\gamma=30$ gives $\epsilon_{0} / E_{s}=0.092$. This is far too large to be practical but sets the scale of the problem. Taking a dispersion of $2 \mathrm{~m}$ and a physical aperture of $3.5 \mathrm{~cm}$ gives an absolute maximum momentum spread of \pm 0.0175 . For $\lambda=1$ and $\eta=10^{-4}, V_{s}=431 \mathrm{~V}$. With this synchronous voltage the total acceleration time would be $\sim 28$ times longer than the $28 \mathrm{MHz}$ scenario.

$$
\begin{gathered}
V_{s}=\frac{\eta\left(E_{s} / q\right)}{2 \lambda} \frac{\hat{\epsilon}^{2}}{E_{s}^{2}} \\
\frac{d \gamma}{d t}=\frac{V_{s}}{\left(m c^{2} / q\right) T_{r e v}}=\frac{1}{\left(m c^{2} / q\right) T_{r e v}} \frac{\eta\left(E_{s} / q\right)}{2 \lambda} \frac{\hat{\epsilon}^{2}}{E_{s}^{2}}
\end{gathered}
$$

For an acceleration $\dot{\gamma}$ the synchronous particle with energy $E_{s}$ experiences a net voltage per turn given by $V_{s}=\left(m c^{2} / q\right) \dot{\gamma} / f_{\text {rev }}$. For protons with $\dot{\gamma}=1 s^{-1}, V_{s}=12 \mathrm{kV}$.

One solution to the problem is to provide an RF voltage that cancels $V_{s}$ within the bunch. Taking a bunching factor of $1 / 2$ a square wave rf voltage with a period of $78 \mathrm{kHz}$ and a voltage of $12 \mathrm{kV}$ would work. One can imagine schemes using a broad band cavity and amplifier as employed in induction accelerators but the duty cycle requirements in RHIC are much more severe. Developing such a system would be a major R\& D effort. In Refs. [3,4] induction devices are discussed for acceleration. These may be an alternative solution.

\section{Summary}

We investigated the effect of bunch length and crossing angle on the achievable luminosity in RHIC, given a limit for the incoherent beam-beam spread of $\Delta Q_{\max }=-0.03$. We considered bunches in the storage rf system, in the accelerating rf system and superbunches that fill the ring except for an abort gap. 
Crossing angles of larger than 5 mrad are needed to gain from long high intensity bunches. 5 mrad is the existing limit for vertical crossing angles at storage energy. Larger horizontal angles can be implemented.

Very high luminosities can be achieved with superbunches. While an abort gap can be maintained with an barrier cavity, the beam cannot be accelerated with such an rf system. Acceleration may be based on induction devices. In addition, the trigger systems of the experiments have to be changed in a fundamental way.

The beam-beam limitation considered in this article is only the incoherent tune spread. Odd order and synchro-betatron resonances that are driven with crossing angles are ne-

glected, so are collective effects. A substantial rise in the beam current would also be limited by other effects, and changes in a number of systems will be needed [13].

\section{Acknowledgements}

The authors are thankful for discussions with M. Brennan, M. Harrison, W. MacKay, T. Roser, F. Ruggiero, S. Peggs, V. Ptitsyn, and F. Zimmermann.

\section{References}

[1] E. Keil, "Luminosity optimization for storage rings with low- $\beta$ sections and small crossing angles", Nucl. Instrum. Methods 113, 333 (1973).

[2] F. Ruggiero and F. Zimmermann, "Luminosity optimization near the beam-beam limit by increasing bunch length or crossing angle", Phys. Rev. ST Accel. Beams 5061001 (2002).

[3] K. Takayama, J. Kishiro, M. Sakuda, Y. Shimosaki, and M. Wake, "Superbunch hadron colliders", Phys. Rev. Lett. Vol. 88 No. 14 (2002).

[4] J. Kishiro and K. Takayama, "Circular induction acclerators", KEK preprint 98-210 (1998).

[5] D. Barton, PHOBOS; W. Cristie, STAR; M. Purschke, PHENIX; D. Beavis, BRAHMS; private communication (2002)

[6] S. Peggs, V. Ptitsin, S. Tepikian, P. Thompson, and D. Trbojevic, "Interaction region closed orbits", BNL RHIC/AP/135 (1997).

[7] V. Ptitsyn, private communication (2002).

[8] W. MacKay, "Luminosity as calculated from machine parameters", BNL C-A/AP/89 (2002).

[9] W. Schnell, "Report on the ISR", proceedings of the 1975 Particle Accelerator Conference, Washington, D.C. (1975). 
[10] K.Y. Ng, "Multiple Injections with Barrier Buckets", proceedings of the 1998 European Particle Accelerator Conference, Stockholm, Sweden (1998).

[11] M. Blaskiewicz, J.M. Brennan, T. Roser, K. Smith, R. Spritz, A. Zaltsman, M. Fujieda, Y. Iwashita, A. Noda, M. Yoshii, Y. Mori, C. Ohmori, Y. Sato, "Barrier Cavities in the Brookhaven AGS", proceedings of the 1999 Particle Accelerator Conference, New York (1999).

[12] T. Bohl, T. Linnecar and E. Shaposhnikova, "Barrier buckets in the CERN SPS", proceedings of the 2000 European Particle Accelerator Conference, Vienna, Austria (2000).

[13] M. Harrison, "High luminosity p-p operation at RHIC", BNL RHIC/AP/8 (1993). 